\title{
Optical Light Curves of the Black Hole Binaries GRS 1124-68 and A0620-00 in Outburst: The Importance of Irradiation
}

\section{Citation}

Esin, Ann A., Erik Kuulkers, Jeffrey E. McClintock, and Ramesh Narayan. 2000. “Optical Light Curves of the Black Hole Binaries GRS 1124-68 and A0620-00 in Outburst: The Importance of Irradiation." The Astrophysical Journal 532 (2): 1069-77. https://doi.org/10.1086/308615.

\section{Permanent link}

http://nrs.harvard.edu/urn-3:HUL.InstRepos:41384928

\section{Terms of Use}

This article was downloaded from Harvard University's DASH repository, and is made available under the terms and conditions applicable to Other Posted Material, as set forth at http:// nrs.harvard.edu/urn-3:HUL.InstRepos:dash.current.terms-of-use\#LAA

\section{Share Your Story}

The Harvard community has made this article openly available.

Please share how this access benefits you. Submit a story.

Accessibility 
To Appear in The Astrophysical Journal

Preprint typeset using LATEX style emulateapj v. 04/03/99

\title{
OPTICAL LIGHTCURVES OF THE BLACK HOLE BINARIES GRS 1124-68 AND A0620-00 IN OUTBURST: THE IMPORTANCE OF IRRADIATION
}

\author{
ANN A. $\operatorname{Esin}^{1}$ \\ Caltech 130-33, Pasadena, CA 91125; aidle@tapir.caltech.edu \\ Institute for Theoretical Physics, University of California, Santa Barbara, CA 93106 \\ ERIK KUULKERS \\ Space Research Organization Netherlands, Sorbonnelaan 2, 3584 CA Utrecht, The Netherlands; \\ E.Kuulkers@sron.nl \\ Astronomical Institute, Utrecht University, P.O. Box 80000, 3507 TA Utrecht, The Netherlands \\ AND \\ JefFrey E. MCClintock And RAmesh NARAyAN \\ Harvard-Smithsonian Center for Astrophysics, 60 Garden Street, Cambridge, MA 02138; \\ jmcclintock@cfa.harvard.edu, rnarayan@cfa.harvard.edu \\ To Appear in The Astrophysical Journal
}

\begin{abstract}
We test whether the model proposed by Esin et al. to explain X-ray observations of the black hole soft X-ray transient GRS 1124-68 in outburst can also explain the optical lightcurves of a similar object A0620-00. We show that to reproduce the observed X-ray to optical flux ratio in A0620-00, we need to assume X-ray irradiation of the outer disk that is significantly in excess of what is expected for a standard planar disk. With enhanced irradiation, the Esin et al. model can reproduce the optical evolution of A0620-00 in outburst very well. Though we find that optical observations of GRS 1124-68 also imply enhanced X-ray heating of the outer disk, the irradiation appears to be a factor of $\sim 3$ weaker in this system than in A0620-00. This is surprising, since GRS 1124-68 has a larger disk. We speculate that enhanced irradiation may be due to disk warping, and that the degree of warping differs between the two binaries.
\end{abstract}

Subject headings: accretion, accretion disks - black hole physics - plasmas - radiation mechanisms: thermal

\section{INTRODUCTION}

A number of known X-ray sources exhibit dramatic outbursts in which their bolometric luminosity increases by many orders of magnitude. A fraction of these systems, termed soft X-ray transients (SXTs), is believed to contain binaries in which a more massive compact star is accreting mass from a less massive late-type companion (see Tanaka \& Shibazaki 1996 and van Paradijs \& McClintock 1995 for the most recent comprehensive reviews, though several new SXTs were discovered in the last few years). This subset of X-ray transients is especially interesting because the low mass of their secondary companions has in several cases allowed us to measure the mass functions of the compact primary to be above $3 M_{\odot}$, thus providing the first (and so far only) unambiguous evidence for the existence of stellar mass black holes (see McClintock 1998 and Charles 1999 for the most up-to-date list of black hole SXTs).

${ }^{1}$ Chandra Fellow
The behavior of black hole SXTs (BHSXT) during their outbursts shows a number of common features. A "typical" lightcurve consists of a fast rise $(\tau \sim$ few days) followed by a longer $(\tau \sim$ month $)$ exponential decline back to the system's quiescent luminosity, sometimes with one or more secondary maxima, though not all BHSXTs follow this standard prescription (see Chen, Shrader, \& Livio 1997 for an excellent inventory of observed lightcurves). The X-ray spectral evolution of these systems during outbursts is less well studied. There are many indications that the majority of sources start out fairly soft near the peak (hence their name), i.e. with the total emission dominated by a component with $T \sim$ few $\mathrm{keV}$, and soften further during the decline, before becoming suddenly very hard (the total energy output is dominated by $\sim 100 \mathrm{keV}$ photons) several months after the peak (e.g. Tanaka \& Lewin 1995; Tanaka \& Shibazaki 1996). However, there are several exceptions; e.g. GS 2023+338 and GRO J0422+32 re- 
mained hard throughout their outburst, though the latter displayed a very typical exponential lightcurve. Very little is known about the X-ray spectra of BHSXTs in quiescence, due mainly to their faintness; only V404 Cyg has a well determined spectrum (see Narayan, Barret \& McClintock 1997).

It is now generally accepted that the outbursts in BHSXTs are caused by a thermal instability in the accretion disk, the same mechanism that is believed to cause outbursts in dwarf novae (see e.g. Cannizzo 1993: Lasota 1996; Cannizzo 1998). This model relies on the fact that in an optically thick accretion disk with a central temperature $T_{c} \sim 10^{4} \mathrm{~K}$ (the ionization temperature of hydrogen) the opacity is a very strong function of $T_{c}$. Both cold neutral disks and hot fully-ionized disks are stable, but when the central disk temperature is close to the critical value, thermal instability forces the disk to undergo a limit cycle. In quiescence the disk is cold and its mass accretion rate is considerably below the mass transfer rate from the companion. In this way the disk accumulates mass until increasing density and temperature force it into a hot ionized state, characterized by a very high (often on the order of $\dot{M}_{\text {Edd }}$ ) mass accretion rate, thereby starting an outburst. Of course this high mass accretion rate can only be sustained until the surface density in the disk is depleted sufficiently to force a transition back into the cold neutral state, after which the system settles back into quiescence.

A detailed numerical model of such an outburst in a BHSXT is currently under construction (see e.g. Dubus, Lasota \& Hameury 1999a; Hameury et al. 1998)]. It is already clear however, that illumination of the outer disk by X-rays produced near the black hole plays a very important role (e.g. van Paradijs \& McClintock 1994; van Paradijs 1996; Lasota 1996). King \& Ritter (1998) in a recent paper plausibly argued that such irradiation would naturally produce a prototypical exponential lightcurve. These authors also attribute the departures from the standard lightcurve shape to the size of the disk, claiming that in larger systems irradiation is unable to heat up the outer disk to temperatures above $10^{4} \mathrm{~K}$. In such a case a cooling wave setting off from the outer disk edge would shut off the mass flow early on, producing a short outburst with a linear decline. This suggestion seems to be supported by observations, as shown by Shahbaz, Charles \& King (1998), as well as by detailed theoretical modeling (Dubus et al. 1999a).

Though the disk instability model outlined above offers a plausible explanation of the general lightcurve shapes seen in outbursts of BHSXTs, the origins of the spectral evolution of these systems are still very much debated in the literature. In an attempt to unify different "spectral states" seen dur- ing the decline from the outburst peak to quiescence, Esin, McClintock, \& Narayan (1997, hereafter EMN) have proposed a model for the prototypical BHSXT GRS 1124-68 (also Nova Muscae 1991). In their scenario, luminous soft spectra (with characteristic photon index $\Gamma \lesssim 2.5$ ) seen near the peak of the outburst, when the system is in the so called Very High and High states (hereafter VHS and HS respectively), are modeled by a cool optically thick accretion disk (Shakura \& Sunyaev 1973) topped with a hot optically thin corona (e.g. Haardt \& Maraschi 1991). In the less luminous Low state (hereafter LS), when the observed spectra are very hard (with $\Gamma \sim 2.0-1.5$ ), as well as in the Quiescent state, the accretion flow assumes a different configuration: a hot and optically thin inner flow (a sort of diskless corona) is surrounded by an optically thick outer disk with an inner edge anywhere between several tens and tens of thousands Schwarzschild radii from the accreting black hole.

EMN model the hot inner part as an optically thin advection-dominated accretion flow (ADAF) discovered by Ichimaru (1977), which has been extensively discussed in the literature in the last six years (see Narayan, Mahadevan, \& Quataert 1998 and Kato, Fukue \& Mineshige 1998 for latest reviews). An ADAF is a hot and stable accretion flow solution alternative to the standard Shakura \& Sunyaev (1973) thin disk. It is characterized by electron temperatures above $10^{9} \mathrm{~K}$ and optical depth $\lesssim 1$, which are ideal conditions for producing the hard spectra seen in the low state through thermal Comptonization of the seed synchrotron and thin disk photons. Since an ADAF solution only exists for mass accretion rates below some critical value, $\dot{M}_{\text {crit }}$, it suggests a natural explanation for the transition from the hot flow + outer thin disk configuration at low $\dot{M}$ to thin disk + corona at $\dot{M}>\dot{M}_{\text {crit }}$.

A model involving an advection-dominated flow at accretion rates well below $\dot{M}_{\text {crit }}$ surrounded by an outer thin disk had already been shown to explain well the puzzling properties of quiescent BHSXTs (e.g. Narayan, McClintock, \& Yi 1996; Narayan et al. 1997; Hameury et al. 1997). In their paper, EMN demonstrated that the scenario outlined above works for systems in outburst; it reproduces very well both soft and hard X-ray lightcurves of GRS 1124-68 as well as the details of its spectral evolution. However, though GRS 1124-68 had excellent X-ray coverage, the optical (e.g. Bailyn 1992; King, Harrison, McNamara 1996; Della Valle, Masetti \& Bianchini 1998) and UV (Shrader \& Gonzalez-Riestra 1993) observations, which are the only diagnostic of the outer disk properties, were limited to the first 150 days of the outburst. At later times EMN could obtain use- 
ful constraints only on the innermost regions of the accretion flow, the properties of which are well constrained by the observed X-ray emission. Particularly unfortunate is the fact that the important High-Low state transition occurred during a period with almost no optical coverage. According to the EMN model, during this transition, the source of the X-ray emission shifted from the flat disk to a quasi-spherical corona, causing a considerable change in the angular distribution of radiation incident onto the outer disk. This effect could potentially be seen in the optical light curve.

Though we cannot remedy the lack of optical coverage for GRS 1124-68, in this paper we attempt to do the next best thing, i.e. we attempt to complement the work of EMN by using the extensive multiwavelength photometry available for the outburst of another BHSXT, A0620-00, to infer the properties of the outer disk in these two systems. The quality of the X-ray data available for A0620-00 (though superior for its time; see Kuulkers 1998) renders it unusable for detailed modeling similar to that done for GRS 1124-68. However, in sections $\S 2$ and $\S 3$ we will demonstrate that the two systems are sufficiently alike in their binary parameters and outburst properties to justify a direct use of the model proposed for GRS 1124-68, with only minor parameter changes.

In 8 and $\$ 5$ we describe the modeling of the optical data and present our results, which are then further discussed in 86 . We conclude with a summary in 8 []

\section{A0620-00 AND GRS 1124-68 AS BINARY SYSTEMS}

The remarkable similarity between GRS 1124-68 and A0620-00 in their orbital parameters Bailyn 1992; Remillard, McClintock, \& Bailyn 1992), appearance of the secondary (Remillard et al. 1992) and of the accretion disk in quiescence (Orosz et al. 1994), has been well documented in the literature. To emphasize how closely these two systems resemble each other, we show in Table 1 a summary of their latest binary parameters, gathered from the literature.

The properties of BHSXT outburst lightcurves depend largely on the mass of the primary, which determines the overall energy scale for the system, mass transfer rate from the companion, and the size of the accretion disk. The latter is very important since it probably dictates the value of the mass accretion rate at the peak of the outburst as well as the overall lightcurve shape (e.g. King \& Ritter 1998). It is clear from Table 1, that the data are consistent with the black holes in GRS 1124-68 and A0620-00 having similar masses. Since their orbital periods are short, this argues that the mass transfer rate cannot be very different in the two systems (e.g. King,
Kolb \& Burderi 1996). Finally, the size of the accretion disk, $R_{\text {out }}$, can be estimated from other orbital parameters, assuming that it fills a fixed fraction of the primary Roche Lobe (we take it to be $80 \%$ ). We compute $R_{\text {out }}$ using the Paczyński (1971) formula:

$$
\begin{aligned}
R_{\text {out }} & =2.82 \times 10^{10} P_{\text {orb }}^{2 / 3}[m(1+q)]^{1 / 3} \\
& \times(0.38-0.2 \log q) \mathrm{cm},
\end{aligned}
$$

where $P_{\text {orb }}$ is the binary orbital period measured in hours, $q=M_{c} / M$ is the mass ratio, and $m=$ $M / M_{\odot}$ is the mass of the black hole in solar units. In calculating accretion disk size for A0620-00 we adopted $m=4.5$ (see \$3) and $q=0.067$, while for GRS 1124-68 we took $m=6$ and $q=0.133$. The resulting values of $R_{\text {out }}$ for both objects with the corresponding uncertainties are shown in Table 1. Since the orbital period of A0620-00 is shorter than that of GRS 1124-68, the accretion disk of the former is somewhat smaller in physical units, though in Schwarzschild units the two disks have roughly the same sizes.

\section{X-RAY OUTBURSTS OF GRS 1124-68 \& A0620-00}

Given that these two binaries resemble each other so closely, it is not surprising that their properties in outburst are similar as well, as we demonstrate below. In Figure 11 (upper panels) we have plotted the observed soft X-ray lightcurves of A0620-00 and GRS 1124-68. Both systems show a fast rise and a characteristic exponential decline after the peak, interrupted by a small secondary and a somewhat larger tertiary maximum. (Though it is not clear from this data that A0620-00 reaches a local maximum near day 200, other instruments showed that the X-ray flux indeed declined shortly after that point, see Kuulkers 1998.) The rates of the exponential decline after the primary maximum in the two objects are very close; for A0620-00 we have $\tau \sim 32$ days, and for GRS $1124-68, \tau \sim 35$ days.

It is considerably more difficult to compare spectral evolutions of the two BHSXTs, since all published spectra of A0620-00 were taken during the first three months after the onset of the outburst. These observations all show fairly soft spectra with characteristic peak temperatures around a few keV (e.g. Carpenter et al. 1976; Citterio et al. 1976; Doxsey et al. 1976; Long \& Kestenbaum 1978) confirming that this system indeed looked like GRS 1124-68 in the VHS and HS. After about $\sim 100$ days, we have to rely on the broadband data, so in Figure 1 (lower panels) we have plotted ratios of count rates in the hard and soft X-ray bands for both objects. The figure shows a nearly identical slow decrease of the hardness ratios for both systems, followed by an abrupt hardening just prior to the rise to the tertiary maximum. The 
Esin et al.

latter feature suggests that a High-Low state transition is taking place.
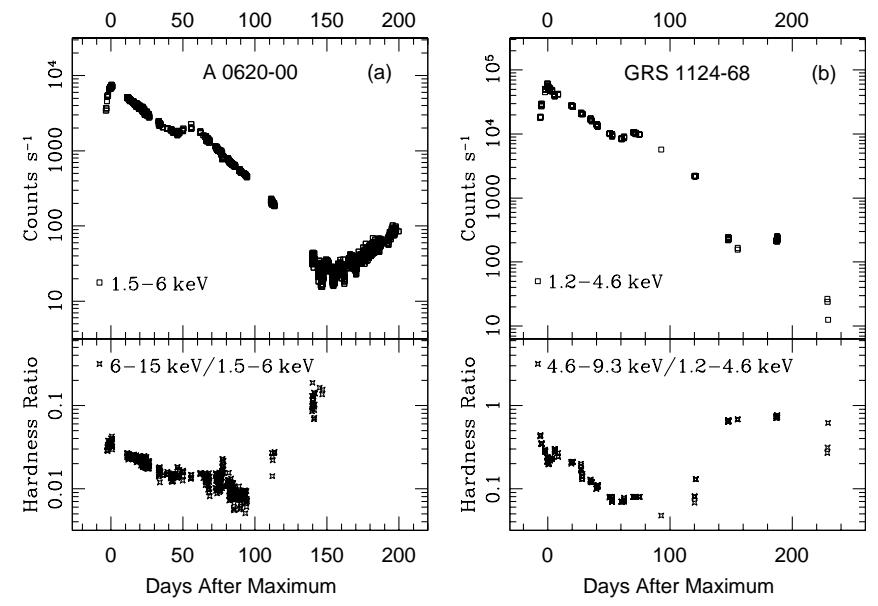

Fig. 1.- (a) Soft X-ray lightcurve and hardness ratio of A0620-00 obtained with SAS-3 CSL A $(1.5-6 \mathrm{keV})$ and CSL B $(6-15 \mathrm{keV})$ detectors (courtesy K. Plaks, J. Woo and G. Clark; adopted from Kuulkers 1998). (b) Soft X-ray lightcurve and hardness ratio of GRS 1124-68 obtained with Ginga LAC detectors (courtesy of K. Ebisawa). Note that error bars are not plotted in either panel.

So far in our comparison of the X-ray lightcurves and hardness ratios we used raw count rates, since this is the information most readily available for A0620-00. Though in general a questionable procedure, we believe it is justified here because Ginga $L A C$ and $S A S$ - 3 contained detectors with very similar responses in the energy bands of interest (Buff et al. 1977; Turner et al. 1989). We conclude that the existing data strongly supports our hypothesis that the X-ray outbursts of these two BHSXTs showed almost identical evolution. However, our goal is to adapt the EMN model to A0620-00, and since we cannot convert the existing A0620-00 data to physical flux units (or at least not enough of it to be of use) for comparison with the model, we have to obtain some sort of quantitative mapping between GRS 1124-68 and A0620-00 lightcurves. If this can be done, then we can assume that the model for GRS 1124-68 (with properly adjusted parameters, as deduced from the lightcurve mapping) reproduces the behavior of A0620-00 in the X-ray band and proceed to test it against the optical data.

EMN adopted the following mapping relation between the time after the outburst maximum ( $T$, measured in days), mass accretion rate $(\dot{M})$ and the transition radius between the inner hot flow (ADAF) and the outer thin disk $\left(R_{\mathrm{tr}}\right)$ :

$$
T=\tau_{\dot{M}} \ln \left(\frac{\dot{M}_{\text {peak }}}{\dot{M}}\right)+\tau_{R_{\mathrm{tr}}} \ln \left(\frac{R_{\mathrm{tr}}}{3 R_{\mathrm{g}}}\right) .
$$

At $T=0$, mass accretion rate is at its maximum value of $\dot{M}_{\text {peak }}$ and the transition radius is at its minimum value of $3 R_{\mathrm{g}}$ (the marginally stable orbit for a nonrotating black hole), i.e. most of the mass is accreting via a thin disk. As $T$ increases, the mass accretion rate decreases exponentially (with a characteristic efolding time scale $\tau_{\dot{M}}$ ) as the disk empties out, as argued by King \& Ritter (1998). In this regime, a dominant soft component originates in the thin disk and hard X-ray emission comes from a hot corona above the disk. According to the relative importance of the hard X-ray component, EMN identified the resulting spectra with the VHS (stronger high energy emission) or HS (very weak high energy emission).

Once $\dot{M}$ drops below the critical value for the formation of an ADAF, $\dot{M}_{\text {crit }}$, the disk begins to evaporate starting from its inner edge, forming the hot accretion flow and thereby increasing the transition radius (on an e-folding time scale $\tau_{R_{\mathrm{tr}}}$ ). This is accompanied by a dramatic change in the X-ray spectrum. Since now most of the emission originates in the very hot, rarefied gas filling the region inside $R_{\mathrm{tr}}$, the spectrum quickly becomes very hard, with most of the emission coming out in $\sim 100 \mathrm{keV}$ photons, which are produced via inverse Compton scattering of seed synchrotron and disk photons. This regime, when $\dot{M}$ through the inner region stays near its critical value (fueled by the evaporation of the disk, see $\S(6$ ) and $R_{\mathrm{tr}}$ increases with time, was identified by EMN with the Intermediate state (hereafter IS). In this state, spectral and temporal properties of BHSXTs shift from those characteristic of the HS (soft spectrum) to those of the LS (hard spectrum). Finally, at some point (perhaps when $R_{\mathrm{tr}}$ reaches its value in quiescence) mass accretion rate through the inner region begins to drop again and the system moves from the LS into quiescence.

In general, there is no reason to expect the parameters in equation (3-1) to be exactly identical for two systems, even as otherwise similar as those considered in this paper. However, in the scenario proposed by EMN, there is one quantity which must remain relatively constant from system to system, and that is $\dot{m}_{\text {crit }}=\dot{M}_{\text {crit }} / \dot{M}_{\text {Edd }}$, where $\dot{M}_{\text {Edd }}=$ $1.39 \times 10^{18} \mathrm{~m} \mathrm{~g} \mathrm{~s}^{-1}$ is the Eddington mass accretion rate for a black hole of mass $m=M / M_{\odot}$. Therefore, we expect that in Eddington units the X-ray luminosity of BHSXTs in the IS is more or less constant.

Based on this fundamental assumption, in Figure 2 we have plotted soft X-ray lightcurves for our two BHSXTs, where the A0620-00 flux was scaled to obtain the best match in the section of the lightcurves corresponding to the intermediate state, as marked on the figure. The fluxes are normalized to Crab units to remove the effect of different instrumental effective areas. Then the scaling factor $\eta=F_{A} / F_{G R S}=8$ 
must be due only to the difference in the black hole masses, binary inclinations and distances to the two systems. Roughly, we have

$$
\eta=\frac{F_{A}}{F_{G R S}} \approx\left(\frac{m_{A}}{m_{G R S}}\right)\left(\frac{\cos i_{A}}{\cos i_{G R S}}\right)\left(\frac{d_{G R S}}{d_{A}}\right)^{2} .
$$

Note that though the dependence of the observed flux on $\cos i$ used here is strictly valid only for an optically thick disk, the disk emission dominates soft X-ray flux during early part of the IS. Moreover, near $\dot{m}_{\text {crit }}$ the emission from ADAF also escapes preferentially along the polar axes, where the gas density is lower (see Narayan \& Yi 1995 and Narayan et al. 1997).

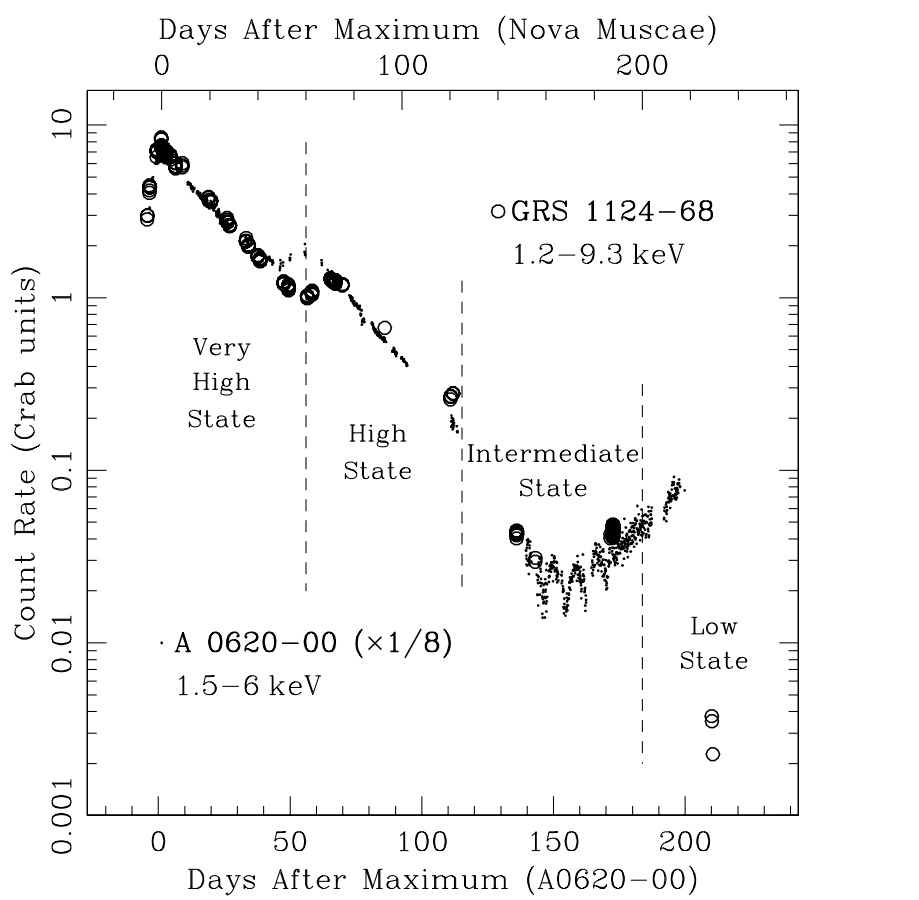

Fig. 2.- Soft X-ray lightcurves of A0620-00 (SAS-3 CSL A; $1.5-6 \mathrm{keV}$ ) and GRS 1124-68 (Ginga LAC $1.2-$ $9.3 \mathrm{keV}$ ) plotted in Crab units. Different spectral states seen during the outburst of GRS 1124-68 are indicated in the figure. The count rate for A0620-00 has been multiplied by a factor of $1 / 8$, to match the curves in the IS, when, according to the EMN model, mass accretion rate is on the order of $\dot{m}_{\text {crit }}$. Note the close correspondence between the lightcurves. They differ only in the time of the secondary maximum and in the width of the tertiary peak.

EMN adopted $m_{G R S}=6, i_{G R S}=60^{\circ}$ and $d_{G R S}=$ $5 \mathrm{kpc}$ and showed that their model reproduces the X-ray data of GRS 1124-68. Given these values for GRS 1124-68 and the constraints on the distance to A0620-00 (see Table 1), equation (3-2) favors a somewhat low black hole mass for A0620-00. For $m_{A}=4.5$, we obtain $d_{A}=1.4 \mathrm{kpc}$ or $1.5 \mathrm{kpc}$, depending on the value of the $f(M)$. Larger values of $m_{A}$ require placing A0620-00 at an even greater distance, apparently inconsistent with existing observations. An even smaller black hole mass in A0620-00, e.g. $m_{A}=3.5$, is formally consistent with all the data; however, there is a preference towards a somewhat higher value. Therefore, in our model for A0620-00 we adopt the largest black hole mass that is still consistent with the distance to the system: $m_{A}=4.5 ; i_{A}=65^{\circ} ; d_{A}=1.4 \mathrm{kpc}$.

The values of the three parameters for A0620-00 introduced in equation (3-1) can be read off Figure 2. By a direct comparison of the two outburst time scales, we obtain $\tau_{\dot{M}}=32$ days, quite close to $\tau_{\dot{M}}=35$ days for GRS 1124-68. Assuming that at day $200 R_{\mathrm{tr}}=10^{3.9} R_{\mathrm{g}}$ (determined from observations of $\mathrm{H}_{\alpha}$ line in quiescence, see Narayan et al. 1996 and references therein), where $R_{\mathrm{g}}=2.95 \times 10^{5} \mathrm{~m} \mathrm{~cm}$ is the Schwarzschild radius, the length of the intermediate state implies $\tau_{R_{\mathrm{tr}}}=10$ days (as compared with $\tau_{R_{\mathrm{tr}}}=8$ days for GRS 1124-68). Finally, the ratio of the peak luminosity to that in the IS is the same in both systems, which implies that the peak mass accretion rate in A0620-00 was the same as in GRS $1124-68$, i.e. $\dot{m}_{\text {peak }}=\dot{M}_{\text {peak }} / \dot{M}_{\text {Edd }}=3$. Incidentally, similarity of $\dot{m}_{\text {peak }}$ in these two systems suggests that like GRS 1124-68, A0620-00 was in the VHS near the peak of the outburst. The available spectral information, though limited, also supports this conclusion.

One must keep in mind however, that our assumption of constant $\dot{m}_{\text {crit }}$ has been demonstrated to be valid (in theory) only for accreting black holes with zero spin; and it is possible that the value of $\dot{m}_{\text {crit }}$ depends on $a / M$. If the black holes in A0620-00 and GRS 1124-68 have very different spins, the procedure of matching the two lightcurves described above may not be valid. On the other hand, Zhang, Cul \& Chen (1997) and Cui, Zhang \& Chen (1998)] argue that GRS 1124-68 does not contain a rapidly spinning black hole, and since A0620-00 shows similar $\mathrm{X}$-ray spectra, it is probably not likely to have much spin either.

\section{MODELING UBV LIGHTCURVES OF A0620-00}

The model of EMN underpredicted the optical flux of GRS $1124-68$ in the VHS and HS by a factor $\sim 3$, though outer disk irradiation was self-consistently included in their calculations. This is not surprising, since it has recently been demonstrated in several papers (e.g. see Dubus et al. 1999b and references therein) that the standard Shakura \& Sunyaev (1973) thin disks do not provide enough flaring at the outer edge to reproduce the observed levels of irradiation. In fact, self-consistent disk models show that the outer parts of the disk (even when irradiation is taken into account in the disk height calculations) are convex, rather than concave (Tuchman, Mineshige, \& Wheeler 1990; Dubus et al. 1999b), i.e. 
Esin et al.

they are shielded from the X-rays emitted by the inner disk. Since in the luminous spectral states (VHS and HS) most of the X-ray emission originates in the thin disk, rather than the corona, this places very severe onstraints on how important irradiation can be in planar disks.

As we will demonstrate below, the ratio of the optical to X-ray fluxes in A0620-00 is even greater than that in GRS 1124-68. Clearly then, in order to reproduce the bsered optical emission from the for

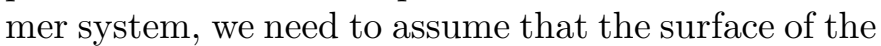
outer disk is considerably more curved than predicted by the standard model. An alternative explanation, involving an extended hot corona which can illuminate even a concave outer disk, cannot apply when the X-ray spectrum is dominated by the disk emission and fails to explain the statistics of disk eclipses in low-mass X-ray binaries (de Jong, van Paradijs \& Augusteijn 1996).

The well-known equation describing irradiating flux at radius $R$ due to a compact central source of $\mathrm{X}$-rays is given by (e.g. Shakura \& Sunyaev 1973; van Paradijs 1996; King \& Ritter 1998):

$$
F_{\text {irr }}(R)=\frac{n L_{\mathrm{X}}(1-a)}{4 \pi R^{2}}\left(\frac{H}{R}\right)^{n}\left[\frac{d \ln H}{d \ln R}-1\right],
$$

where $L_{\mathrm{X}}$ is the total central X-ray luminosity, $a$ is the disk albedo, and $H$ is the height of the disk photosphere above the orbital plane. The power index $n$, in roduced by King \& Ritter (1998), is equal to 1 when the irradiating source radiates isotropically (e.g. as in the case of a corona); and $n$ is set to 2 when the outer disk is irradiated by the inner disk, which emits primarily in the direction perpendicular to the disk surface. The factor of $n$ in the numerator (significant only in the case when $n=2$ ) is necessary to ensure the correct normalization of the total emission from the thin disk.

We know $L_{\mathrm{X}}$ from analyzing the X-ray data (see \$(3), which also allows us to distinguish whether $n=1$ or $n=2$ (or a combination of both) is applicable. Unfortunately, the other two parameters, $H(R)$ and $a(R)$, are not well known. The former because, as we argued above, the standard answer cannot reproduce the observations. The latter because published theoretical calculations of X-ray irradiation, from which a value of $a$ can be derived for a given incident spectrum, generally average over all incident angles (e.g. Magdziarz \& Zdziarski 1995; Zycki et al. 1994: White, Lightman \& Zdziarski 1988), while here only grazing incidence X-rays are relevant. In fact, the values of $a$ inferred from observations of persistent low-mass X-ray binaries with neutron star primaries (de Jong et al. 1996; Kallman, Raymond \& Vrtile $\mathrm{k}$ 1991) are very close to unity, quite different from the generally accepted range, $a=0.1-0.2$

We simplify the problem by lumping the two uncertainties together as a single irradiation parameter $\mathcal{C}(R)$ (Dubus et al. 1999b; Esin, Lasota \& Hynes $1999)$, so that

$$
F_{\text {irr }}=\mathcal{C}(R) \frac{L_{\mathrm{X}}}{4 \pi R^{2}} .
$$

(Note that our $\mathcal{C}$ is defined through $L_{\mathrm{X}}$, while Dubus et al. (1999b) define their parameter through $M c^{2}$, so that the two differ by the factor $\eta=0.1$, which represents the efficiency of converting mass into X-rays for the thin disk.) Moreover, we assume that the only radial dependence of $\mathcal{C}$ comes from $H(R)$, for which we adopt a rather ad hoc prescription: $H \propto R^{9 / 7}$. In systems in outburst, the optical band generally falls in the Rayleigh-Jeans regime of the outermost disk radius. Therefore, the total optical emission is well described simply by the value of $H$ at the outer disk edge, $H\left(R_{\text {out }}\right)$, and the exact form of $H(R)$ affects only the details of the optical spectra. We picked the prescription above derived for isothermal disks (Vrtilek et al. 1990), solely to make the connection with previous literature on disk irradiation, though we know that the outer disks in A0620-00 and GRS 1124-68 are not isothermal. For the X-ray albedo we adopt (simply for lack of anything more appropriate) the angle averaged prescription for the X-ray irradiation of a cold unionized medium (White et al. 1988) which gives $a \sim 0.1-0.2$, depending on the spectral distribution of incident photons. We believe that this is not a bad approximation, since grazing incidence irradiation is not likely to increase the value of the albedo above $\sim 0.4$, unless the outer disk has an ionized atmosphere with optical depth of order unity (D. Psaltis, private communication; see also Basko, Sunyaev \& Titarchuk 1974). Thus the error in the quantity $(1-a)$ in Eq. (4-1) is at most $50 \%$, and not an order of magnitude, as implied by the albedo values derived by de Jong et al. (1996). Nevertheless, because of the uncertainty in this quantity, the values for $H\left(R_{\text {out }}\right)$ quoted below cannot be taken at face value; they must simply be interpreted as a measure of $\mathcal{C}$.

With the prescription for the treatment of outer disk irradiation outlined above, we use the EMN model for X-ray emission to compute a series of spectra following the evolution of A0620-00 during the decline phase of its outburst. Throughout the calculation the shape of the outer thin disk was held fixed, and the height of the disk at the outer edge, $H\left(R_{\text {out }}\right) / R_{\text {out }}$, was adjusted to fit the overall normalization of the observed optical emission. The irradiating flux originating in the ADAF was computed using the ray tracing procedure described in detail in 
EMN which is roughly equivalent to using Eq. (4-1) with $n=1$. We calculated the irradiating flux emitted in the inner parts of the thin disk using Eq. (4-1) with $n=2$. To describe the emission from the thin disk we used the standard modified black-body spectrum. The effective temperature at each radius was computed by equating the black-body emission to the sum of the internal energy dissipation rate in the disk and the heating rate due to irradiation (as described in EMN). Note that in the outer disk, which is primarily responsible for the observed optical emission, $\mathrm{X}$-ray irradiation of the disk surface completely dominates over the viscous heating.

Optical Lightcurves of A0620-00

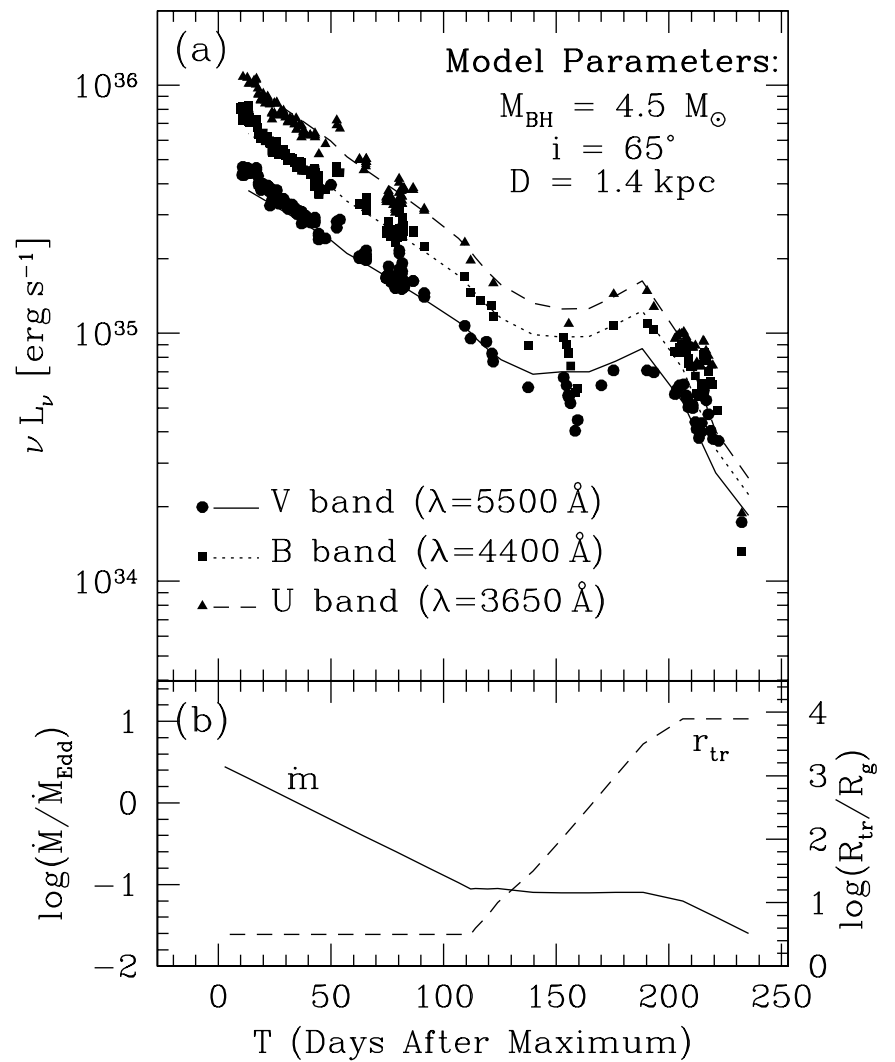

FIG. 3.- (a) The observed specific luminosity of A0620-00 in the three optical bands (Webbink 1978; Kuulkers 1998) is plotted as solid symbols together with the best fitting model lightcurves, obtained with $H\left(R_{\text {out }}\right)=0.12 R_{\text {out }}$. Note that most of the optical flux was generated by the reprocessing in the outer disk of the X-rays emitted by the inner disk and the corona (in the VHS and HS) or by the ADAF (in the IS and LS). (b) The variation of the mass accretion rate $\dot{m}=\dot{M} / \dot{M}_{\mathrm{Edd}}$ and the transition radius $r_{\mathrm{tr}}=R_{\mathrm{tr}} / R_{\mathrm{g}}$ with time in the model.

The resulting optical lightcurves in the standard UBV optical bands, corresponding to the best fitting value of $H\left(R_{\text {out }}\right)=0.12 R_{\text {out }}$ are shown in Figure 3(a). In the same figure we plotted the observed optical fluxes for A0620-00 (from Webbink 1978 and Kuulkers 1998), dereddened for $E_{B-V}=0.35$ (Wu et al. 1983) and converted to luminosity values assuming the distance to A0620-00 of $1.4 \mathrm{kpc}$.

As in modeling the X-ray data, we used the mapping given by equation (3-1) to construct our lightcurves. Figure 3(b) shows how two main model parameters, $\dot{m}=\dot{M} / \dot{M}_{\text {Edd }}$ and $r_{\mathrm{tr}}=R_{\mathrm{tr}} / R_{\mathrm{g}}$, change with time during the decline phase of the outburst. The transition radius changes only during the IS, when mass accretion rate stays constant. Before and after the IS, $\dot{m}$ decreases exponentially with time. Here we take the value of $r_{\mathrm{tr}}$ at the end of the IS to be the same as that suggested by observations in quiescence (Narayan et al. 1996). There is no direct observational support for this assumption from the studies of X-ray spectra, since the shape of X-ray emission stays essentially fixed for any value of $r_{\mathrm{tr}}$ above $\sim 30$ (see e.g. Esin et al. 1998). However, a comparison of mass accreted during the IS with the total mass stored in the thin disk (see $\$$ 6) favors a large change in $r_{\mathrm{tr}}$.

The general correspondence between the model lightcurves and the data in Figure 3(a) is excellent. Taking into account the quality of the EMN fit to GRS 1124-68 X-ray data, one cannot expect to produce optical lightcurves based on this model that would be in better agreement with observations.

Two points are important to emphasize. First, we reproduce the slower decay of the optical intensity $\left(\tau_{\text {opt }} \sim 75\right.$ days $)$ relative to the X-ray intensity ( $\tau_{\mathrm{x}} \sim 25$ days $)$, which is a typical feature of optical lightcurves in BHSXTs. Though optical emission comes purely from X-ray irradiation, cooling of the outer disk, which accompanies a decline in the irradiating flux, causes the peak of the emission to shift towards the optical band. This effect compensates for the total decline in the reprocessed emission and considerably increases the e-folding time of the observed optical flux (see also the discussion in King \& Ritter 1998).

The second feature of the observed lightcurves which is reproduced well in the model, is the upturn during the IS (between days 130 and 200). In our model this is due to the change in the character of the irradiating flux. During the early parts of the outburst (VHS and HS) X-ray emission comes primarily from the accretion disk, so that the irradiating flux is proportional to the square of the disk height (see Eq. 4-1). During this period the irradiation parameter in our calculations is $\mathcal{C}\left(R_{\text {out }}\right) \simeq 0.004$. On the other hand, in the LS, when all the X-rays come from the isotropically emitting ADAF, the irradiating flux is linear in $H / R$. At this point, we have $\mathcal{C}\left(R_{\text {out }}\right) \simeq 0.03$. During the IS, the value of $\mathcal{C}$ changes gradually between these two limiting values, causing an upturn in the lightcurve. This feature may be an additional 
Esin et al.

confirmation of our assumption that during this part of the outburst the transition radius is increasing.

Though the overall normalization of the model lightcurves is determined by our choice of $H\left(R_{\text {out }}\right)$, it is interesting that the model produces the correct spacing between the $\mathrm{V}, \mathrm{B}$, and $\mathrm{U}$ lightcurves, indicating that our optical band spectra must agree with the data fairly well. However, because the functional form of $H(R)$ was chosen arbitrarily, this may not be a very significant result.

\section{REVISITING GRS 1124-68}

As mentioned above, the original EMN model fell short (by a factor of 3) of reproducing the optical emission from GRS 1124-68. However, with the improved treatment of irradiation we now have no trouble in fitting those data, as demonstrated in Figure 4. The UV data points were taken from Shrader \& Gonzalez-Riestra (1993). The V band data are collected from Bailyn (1992); King et al. (1996); Della Valle et al. (1998); and other observations tabulated by Cheng et al. (1992, see references therein). Cheng et al. also obtained an HST spectrum of the system which we used to estimate the observed flux at $3100 \AA$ for day 127 . All the data points were dereddened using the color index $E_{B-V}=0.30$ quoted by Shrader \& Gonzalez-Riestra (1993). The model lightcurves were calculated using the parameters for GRS 1124-68 given in EMN.

Optical Lightcurves of GRS 1124-68

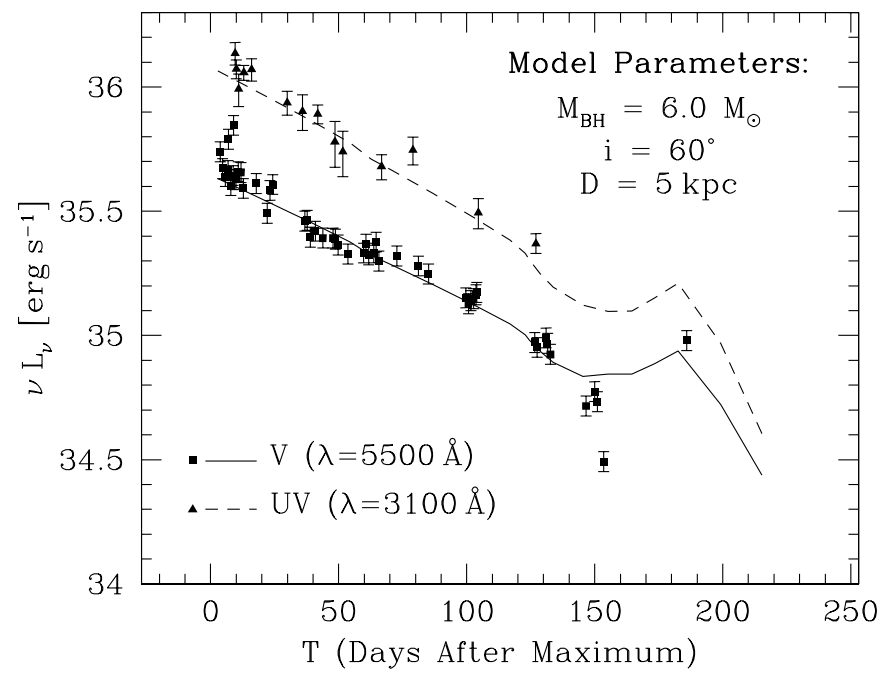

FIG. 4.- Optical and UV photometry for GRS 1124-68 are plotted as solid points. Errorbars on the UV points are quoted by Shrader \& Gonzalez-Riestra (1993), and those on the $\mathrm{V}$ band points are all set to $\pm 0.1 \mathrm{mag}$. The curves show the model lightcurves of GRS 1124-68 calculated for $\tau_{\dot{M}}=35$ days and $\tau_{R_{\mathrm{tr}}}=8$ days (see EMN). The best fit is obtained with $H\left(R_{\text {out }}\right)=0.07 R_{\text {out }}$.

The most interesting point about the fit in Figure 4 is the comparatively small best fit value of the disk height at the outer edge, $H\left(R_{\text {out }}\right)=0.07 R_{\text {out }}$. Using the fact that most of the data were obtained during the period when X-ray emission is dominated by the disk, we estimate $\mathcal{C}\left(R_{\text {out }}\right) \simeq 0.0014$. This value is considerably smaller than that derived for A0620-00 in $\$$. It is easy to see the origin of this discrepancy. A comparison of Figures $3(\mathrm{a})$ and 1 shows that the optical luminosities of the two BHSXTs were nearly identical. However A0620-00 was a factor of $1.6 \mathrm{dim}-$ mer in X-rays (see $\S$ 3), implying that irradiation must have been considerably more important in this system than in GRS 1124-68.

\section{DISCUSSION}

Our results show that in order to reproduce the optical lightcurves of A0620-00 and GRS 1124-68 efficient irradiation of the outer disk is required. The disk height at the outer edge required to fit the observations is a factor of $\sim 2$ smaller than $H / R \gtrsim 0.2$ found by de Jong et al. (1996) for some persistent LMXBs. However, this comparison of $H / R$ values is complicated by the fact that the albedo values used in our calculations were almost an order of magnitude smaller than those derived by de Jong et al. (1996). In addition, we assumed a different angular distribution for the irradiating flux then de Jong et al. A better way to check our results would be through comparing the effective irradiation parameter at the outer disk edge instead of disk height, since it is really the former that determines the importance of irradiation. The results of de Jong et al. (1996) imply $\mathcal{C}\left(R_{\text {out }}\right) \sim 0.002-0.004$, consistent with what we find in the VHS and HS. On the other hand, our calculations imply that in the IS the heating of the outer disk is almost an order of magnitude stronger because of the isotropy of the irradiating X-rays.

The most puzzling result of our investigation is the fact that a smaller disk in A0620-00 is heated considerably more than its larger counterpart in GRS 1124-68. This conclusion is nearly model independent, since it follows simply from comparing the X-ray to optical flux ratios in the two systems. Of course our main assumption that the mass accretion rate during the IS is the same for A0620-00 and GRS 1124-68 may be incorrect, since we do not know precisely what mechanism is driving the evaporation of the thin disk at this stage of the outburst, or whether the two black holes have the same spin. However, even abandoning this fundamental constraint would not reconcile the results for the two systems. If we allow $\dot{m}$ in the IS to be smaller than $\dot{m}_{\text {crit }}$ in A0620-00, X-ray data allows higher $m$ and smaller $i$ for this system (which incidentally are also preferred by observations in quiescence, see Table 11). The size of the disk in A0620-00 would not be 
strongly affected since it depends only weakly on $m$ (see Eq. 2-1), so most of the effect would come from the change in $i$. In a lower inclination system the ratio of optical emission to X-rays is greater, as is observed in A0620-00, but only if X-ray emission is close to isotropic. Thus, this would have some effect in the late IS and LS, where X-ray emission originates in an ADAF, but will not help in the VHS and HS, were X-rays are emitted predominantly in the disk.

Thus, our comparison of A0620-00 and GRS 1124-68 clearly implies that the disk in the former is for some reason more 'curved', though in all other respects the two systems are nearly identical. This suggests that the shape of the disk in a BHSXT may not be entirely predictable for a given set of parameters, or in other words, that it might depend on the past history of the system. The most obvious example of such an effect is disk warping (e.g. Maloney, Begelman \& Pringle 1996). If the outer disks in BHSXTs (and LMXBs in general) are warped, this can explain both high levels of irradiation and variations between otherwise similar sources, like those we have examined here.

One constraint we can place on disk warping comes from expected luminosity fluctuations due to the long-term precession of the warp. For the simplest warp geometry ( $m=1$ mode) we can estimate the ratio of fluxes observed when the warp is facing the observer and when it is facing away from the observer as $\cos (i-\beta) / \cos (i+\beta)$, where $\beta$ is the angle between the normal to the disk surface at the outer edge and the binary axis, and $i$ is the binary inclination. For our prescription $H \propto R^{9 / 7}$, we have $\beta=\tan ^{-1}(d H / d R) \sim 9^{\circ}$; in general for small warp amplitude $\beta$ is of the same order of magnitude as $H / R$. Thus, for our choice of parameters, the optical emission observed from a warped disk in A0620-00 must vary by a factor of $\sim 2$ on the time scale of several months (see e.g. Maloney et al. 1996 for radiation-driven warping), while the emission from GRS 1124-68 should vary by about 50\%. (Note that no eclipses of either the secondary or the central X-ray source are expected in either A0620-00 of GRS $1124-68$, since in both systems $\beta+i \ll \pi / 2$.) This number of course must be treated as only a rough estimate, as it is very sensitive to the exact shape of the disk surface. However, it is interesting to note that both optical and X-ray lightcurves of A0620-00 show 7-8 day oscillations of roughly the right amplitude (though the period seems to be too short for precession) during the IS (e.g. Tsunemi. Mats loka \& Takagishi 1977; Matilsky et al. 1976; see also Figures 2 and $3 \mathrm{a}$ ), and a hint of the same effect can be seen in the optical lightcurve of GRS 1124-68 (Figure 4, see also the discussion in Kuulkers 1998).
The EMN model does not explain the origin of the secondary maximum seen near day 55 in the lightcurves of A0620-00 and around day 70 for GRS 1124-68. Among several possible explanations for this feature that were proposed in the literature (see Cannizzo 1998 and references therein), two scenarios invoke an enhancement in the central mass accretion rate triggered by irradiation of a new mass source. Augusteijn, Kuulkers \& Shaham (1993, see also Chen, Livio \& Gehrels 1993) propose the secondary star as the source of the extra mass; King \& Ritter (1998) invoke the outer disk, initially left cold by the heating wave. Both mechanisms would cause an increase in the lightcurve decay time $\tau_{\mathrm{v}}$ after the peak of the outburst, where $\tau_{\mathrm{v}}$ is essentially of order of the viscous time scale in the outer disk. In A0620-00, a system with a much smaller disk, this time scale should be smaller as well, consistent with what is observed.

The plateau before the tertiary maximum in the X-ray lightcurves, which we identify with the end of the IS, was much more prominent in A0620-00 than in GRS 1124-68. In our model this means that the phase of constant $\dot{M}$ and increasing $R_{\text {tr }}$ lasted roughly $\sim 20$ days longer in A0620-00. An interesting question to consider is why $\dot{M}$ in the X-ray producing region stays constant during this period. One obvious possibility is that this results directly from disk evaporation. We can write down the equation for the mass accretion rate through the central ADAF during the IS:

$$
\dot{M}_{\mathrm{ADAF}}=\dot{M}_{\mathrm{disk}}+\Sigma_{\mathrm{disk}}\left(R_{\mathrm{tr}}\right) 2 \pi R_{\mathrm{tr}} \dot{R}_{\mathrm{tr}} .
$$

This simply states that the mass flow through the $\mathrm{ADAF}$ is equal to the mass flow through the disk plus the rate of mass evaporation from the inner part of the disk. In outburst, we expect $\dot{M}_{\text {disk }}$ to decrease exponentially with time. Then one can show that if $\dot{M}_{\mathrm{ADAF}}$ is kept constant and the surface density profile in the outer disk is taken to be that of a steady-state disk, the transition radius has to increase roughly exponentially with time. Note that we can assume that $\dot{M}_{\mathrm{ADAF}}$ is constant with $R$ and is equal to the central $\dot{M}$, since the accretion timescale in the ADAF region is many orders of magnitude shorter that the timescale on which the quantities on RHS of Eq. (6-1) vary, i.e. the ADAF adjusts practically instantaneously to the changes in the mass inflow at the transition radius.

Though a realistic functional form of $R_{\mathrm{tr}}(t)$ is probably more complicated than a simple exponential adopted in Eq. (3-1) (since it depends on the detailed evolution of the surface density in the outer disk, which can be obtained only from numerical diskinstability models), the main results of our paper 
are not very sensitive to this detail. For our model lightcurves to match optical data for A0620-00, it is sufficient for $R_{\mathrm{tr}}$ to increase in some fashion from $3 R_{\mathrm{g}}$ to $\sim 10^{2}-10^{4} R_{\mathrm{g}}$ during the IS.

If most of the mass accreted onto the black hole during the IS comes from disk evaporation, it is important to compare the mass accreted during this period to the mass stored in the thin disk at the onset of the IS. The former is simply

$$
M_{\mathrm{acc}}=\dot{M}_{\mathrm{crit}} \Delta t_{\mathrm{IS}}
$$

where $\Delta t_{\text {IS }}$ is the duration of the IS. To estimate the mass stored in the disk we make use of the well known fact that during the outburst the disk adopts a quasisteady density profile, so that

$$
M_{\mathrm{disk}}=2 \pi \int_{0}^{R_{\mathrm{q}}} \Sigma\left(\dot{M}_{\mathrm{crit}}, R\right) R d R
$$

where $\Sigma\left(\dot{M}_{\text {crit }}, R\right)$ is given by the standard outer disk formula from Shakura \& Sunyaev (1973), and $R_{\mathrm{q}}$ is the value of the transition radius at the end of the IS. For A0620-00 we get $M_{\text {acc }} \sim 3 \times 10^{24} \mathrm{~g}$ and $M_{\text {disk }} \sim 6 \times 10^{23}\left(R_{\mathrm{q}} / 10^{3.9} R_{\mathrm{g}}\right)^{5 / 4}(0.1 / \alpha)^{4 / 5} \mathrm{~g}$, where $10^{3.9} R_{\mathrm{g}}$ is the value of the transition radius in quiescence (see Narayan et al. 1996). Because these numbers are very approximate, the discrepancy between them is not serious. However, we can safely draw the conclusion that without any other source of mass, during the IS the transition radius must have increased close to its quiescent value.

According to this interpretation, the outer disk contains very little mass at the end of the IS plateau. This is consistent with the sharp drop in the X-ray (see Kuulkers 1998 and references therein) and optical (see Figure 3a) fluxes observed at that point. Moreover, the last set of optical data points in Figure $3(\mathrm{a})$, observed on day 232 , shows that the B band flux was considerably lower than both $\mathrm{V}$ and $\mathrm{U}$ band fluxes (this was originally pointed out by Lyutyi 1976 and Lyutyi \& Shugarov 1979), implying that perhaps the disk was becoming optically thin at this point.

Though the IS plateau was somewhat shorter in GRS 1124-68, the same calculation for this system gives us very similar values: $M_{\text {acc }} \sim 4 \times 10^{24} \mathrm{~g}$ and $M_{\text {disk }} \sim 10^{24}\left(R_{\mathrm{q}} / 10^{3.9} R_{\mathrm{g}}\right)^{5 / 4}(0.1 / \alpha)^{4 / 5} \mathrm{~g}$. These numbers are clearly at odds with the results of Zycki. Done \& Smith (1998) which seem to indicate that in GRS 1124-68 the transition radius was still fairly small at the end of the IS.

\section{CONCLUSIONS}

We have tested whether the model proposed by Esin, McClintock, \& Narayan (1997) to explain the X-ray behavior of GRS 1124-68 during its 1991 outburst can also reproduce the observed optical lightcurves of another BHSXT, A0620-00. Our comparison of the X-ray data for GRS 1124-68 and A0620-00 strongly suggested that these X-ray novae must have followed the same spectral evolution during their outbursts and therefore the scenario developed for GRS 1124-68 could be directly used in modeling A0620-00. We showed that the EMN model modified to include a flared outer disk, can reproduce the shape of the optical lightcurves of A0620-00. Assuming that the shape of the irradiated outer disk remains fixed throughout the outburst, this result provides important evidence in support of our basic scenario of X-ray production, since it relies on the fact that the primary source of $\mathrm{X}$-rays changes from the inner part of the disk in the Very High and High spectral states to the isotropically emitting ADAF in the Intermediate and Low states.

We estimated the strength of irradiation required to reproduce the observed X-ray to optical flux ratio in A0620-00, and found that we need an irradiation parameter (as defined by Eq. 4-2) of $\mathcal{C} \sim 0.004$ when the X-ray production is dominated by the disk, and $\mathcal{C} \sim 0.03$ when the irradiating flux comes from the ADAF. This corresponds to a fixed disk height at the outer edge of $H / R \sim 0.12$, though this number probably should not be taken at face value due to considerable uncertainty in the value of the X-ray albedo of the outer disk. However, for GRS 1124-68 we estimated that the optical data taken during the VHS and HS requires an irradiation parameter a factor of $\sim 3$ smaller than in A0620-00, corresponding to $H / R \sim 0.07$. We speculate that this difference between otherwise nearly identical systems implies that outer disk flaring is caused by warping.

We thank Ken Ebisawa for providing the raw count rates for GRS $1124-68$ as well as the relevant Crab count rates for Ginga LAC. We acknowledge the use of the processed SAS-3 data from Kenneth Plaks, Jonathan Woo and George Clark. AE gratefully acknowledges useful discussions with Kristen Menou, Jean-Marie Hameury and Jean Pierre Lasota, who significantly enhanced her understanding of the disk instability model. This work was supported by NASA through Chandra Postdoctoral Fellowship grant \#PF8-10002 awarded by the Chandra X-Ray Center, which is operated by the SAO for NASA under contract NAS8-39073. RN was supported in part by grant AST 9820686 from the NSF. 


\section{REFERENCES}

Abramowicz, M. A., Chen, X., Kato, S., Lasota, J. P., \& Regev, O. 1995, ApJ, 4 38, L37

Augusteijn. T., Kuulkers, E., \& Shaham, J. 1993, A\&A, 279, L13

Bailyn, C. D. 1992, ApJ, 391, 298

Barret, D., McClintock, J. E., \& Grindlay, J. E. 1996, ApJ, 473,963

Basko, M. M., Sunyaev, R. A., \& Titarchuk, L. G. 1974, A\&A, 31,249

Begelman, M. C. \& Chiueh, T. 1988,ApJ, 332, 872

Buff, J., et al. 1977, ApJ, 212, 768

Cannizzo, J. 1993, in Àccretion Disks in Compact Stellar Systems, ed. J. Wheeler (Singapore: World Scientific), p. 6

Cannizzo, J. K. 1998, ApJ, 494, 366

Carpenter, G. F., Eyles, C. F., Skinner, G. K., Willmore, A. P., \& Wilson, A. M. 1976, MNRAS, 176, 397

Casares, J., Martin, E. L., Charles, P. A., Molaro, P., \& Rebolo, R. 1997, New Astronomy, 1, 299

Charles, P. A. 1999, in "Theory of Black Hole Accretion Disks", eds. M. Abramowicz, G. Bjornsson \& J. Pringle (Cambridge Univ. Press), p. 1

Chen, W., Shrader, C. R., \& Livio, M. 1997, ApJ, 491, 312

Chen, W., Livio, M. \& Gehrels, N. 1993, ApJ, 408, L5

Cheng, F. H., Horne, K., Panagia, N., Shrader, C. R., Gilmozzi, R., Paresce, F., \& Lund, N. 1992, ApJ, 397, 664

Citterio, O., Conti, G., Di Benedetto, P., \& Tanzi, E. G. 1976, MNRAS, 175,35

Cui, W., Zhang, S.-N., \& Chen, W. 1998, ApJ, 492, L53

de Jong, J. A., van Paradijs, J., \& Augusteijn, T. 1996, A\&A, 314,484

Della Valle, M., Jarvis, B. J., \& West, R. M. 1991, Nature, 355, 614

Della Valle, M., Masetti, N., \& Bianchini, A. 1998, A\&A, 329, 606

Doxsey, R. et al. 1976, ApJ, 203, L9

Dubus, G., Lasota, J.-P., Hameury, J.-M. 1999a, in preparation

Dubus, G., Lasota, J.-P., Hameury, J.-M., Charles, P. A. 1999b, MNRAS, 303, 139

Esin, A. A., Lasota, J.-P., \& Hynes, R. 1999, submitted to MNRAS

Esin, A. A., McClintock, J. E., \& Narayan, R. 1997, ApJ, 489, $865(\mathrm{EMN})$

Esin, A. A., Narayan, R., Cui, W., Grove, J. E., Zhang, S.-N. 1998, ApJ, 505, 854

Haardt, F. \& Maraschi, L. 1991, ApJ, 380, 51

Hameury, J.-M., Lasota, J.-P., McClintock, J. E., Narayan, R. 1997, ApJ, 489, 234

Hameury, J.-M., Menou, K., Dubus, G., Lasota, J.-P., Hure, J.-M. 1998, MiNRAS, 298, 1048

Haswell, C. A., Robinson, E. L., Horne, K., Stiening, R. F., \& Abbott, T. M. C. 1993, ApJ, 411, 802

Ichimaru, S. 1977, ApJ, 214, 840

Kallman, T. R., Raymond, J. C., \& Vrtilek, S. D. 1991, ApJ, 370,717

Kato, S., Fukue, J., \& Mineshige, S. 1998, Black-Hole Accretion Disks (Kyoto: Kyoto Univ. Press)

King, N. L., Harrison, T. E., McNamara, B. J. 1996, AJ, 111, 1675

King, A. R., Kolb, U., \& Burderi, L. 1996, ApJ, 464, L127

King, A. R. \& Ritter, H. 1998, MNRAS, 293, L42

Kitamoto, S., Tsunemi, H., Miyamoto, S., \& Hayashida, K. 1992, ApJ, 394, 609

Kuulkers. E. 1998, New Astronomy Reviews 42, 1 astro$\mathrm{ph} /$ 8005031)

Lasota, J.-P. 1996, in Proceedings of IAU Symposium 165, p. 43

Lasota, J.-P. 1999, to appear in "Disk Instabilities in Close Binary Systems - 25 years of the Disk-Instability Model", eds. S. Mineshige, J. C. Wheeler (astro-ph/9901297)
Lasota, J. P., Narayan, R., \& Yi, I. 1996, A\&A, 314, 813

Long, K. S. \& Kestenbaum, H. L. 1978, ApJ, 226, 271

Lyutyi, V. M. 1976, SvAL, 2, 156

Lyutyi, V. M. \& Shugarov, S. Yu. 1979, SvAL, 5, 102

Magdziarz, P., Zdziarski, À. A. 1995 MNRAS, 273, 837

Maloney, P. R., Begelman. M. C., \& Pringle, J. E. 1996, ApJ, 472,582

Marsh, T. R., Robinson, E. L, \& Wood, J. H. 1994, MNRAS, 266,137

Matilsky, T., Bradt, H. V., Buff, J., Clark, G. W., Jernigan, J. G., Laufer, B., McClintock, J., Zubrod, D., Joss, P. C. 1976, ApJ, 210, L127

McClintock, J. E. 1998, in Accretion Processes in Astrophysical Systems, Proceedings of the 8th Annual Astrophysics Conference in Maryland, eds. S.S. Holt \& T. Kallman (NY: AIP), p. 290

McClintock, J. E. \& Remillard, R. A. 1986, ApJ, 308, 110

Mineshige, S. \& Wheeler, J. C. 1989, ApJ, 343, 241

Narayan, R., Barret, D., \& McClintock, J. E. 1997, ApJ, 482, 448

Narayan, R., Mahadevan, R., \& Quataert, E. 1998, in The Theory of Black Hole Accretion Disks, eds. M. A. Abramowicz, G. Björnsson, \& J. E. Pringle, in press

Narayan, R., McClintock, J. E., \& Yi, I. 1996, ApJ, 457, 821

Narayan, R. \& Yi, I. 1995, ApJ, 444, 231

Oke, J. B. 1977, ApJ, 217, 181

Oke, J. B. \& Greenstein, J. L. 1977, ApJ, 211, 872

Orosz, J. A., Baylin, C. D., Remillard, R. A., McClintock, J. E., \& Foltz, C. 1994, ApJ, 436, 848

Paczyński, B. 1971, ARA\&Ä, 9183

Remillard, R. A., McClintock, J. E., \& Bailyn, C. D. 1992, ApJ, 399, L145

Shahbaz, T., Charles, P. A., \& King, A. R. 1998, MNRAS, 301, 382

Shahbaz, T., Naylor, T., \& Charles, P. A. 1994, MNRAS, 268, 756

Shahbaz, T., Naylor, T., \& Charles, P. A. 1997, MNRAS, 285, 607

Shahbaz, T., Bandyopadhyay, R. M., \& Charles, P. A. 1999, A\&A, 346, 82

Shakura, N. I. \& Sunyaev, R. A. 1973, A\&A, 24, 337

Shrader, C. R. \& Gonzalez-Riestra, R. 1993, A\&A, 276, 373

Tanaka, Y. \& Shibazaki, N. 1996, ARA\&A, 34, 607

Tanaka, Y. \& Lewin, W. H. G. 1995, in X-ray Binaries, eds. W. H. G. Lewin et al., Cambridge Univ. Press., p. 126

Tsunemi, H., Matsuoka, M., \& Takagishi, K. 1977, ApJ, 211. L5

Tuchman, Y., Mineshige, S., \& Wheeler, J. C. 1990, ApJ, 359, 164

Turner, M. J. L., et al. 1989, PAS.J, 41, 345

van Paradijs, J. 1996, ApJ, 464, L139

van Paradijs, J. \& McClintock, J. E. 1994, A\&A, 290, 133

van Paradijs, J. \& McClintock, J. E. 1995, in X-ray Binaries, eds. W. H. G. Lewin et al.. Cambridge Univ. Press., p. 126

Vrtilek, S. D., Raymond, J. C., Garcia, M. R., Verbunt, F., \& Hasinger, G. 1990, A\&A, 235, 162

Webbink, R. F. 1978, "A provisional Optical Light Curve of the X-ray Recurrent Nova V616 Mon = A 0620-00," unpublished report

White, T. R. Lightman, A. P. \& Zdziarski, A. A. 1988, ApJ, 331,939

Wu, C.-C., Panek, R. J., Holm, A. V., Schmitz, M. 1983, PASP, 95,391

Zhang, S.-N., Cui, W., \& Chen, W. 1997, ApJ, 482, L155

Życki, P. T., Done, C., \& Smith D. A. 1998, ApJ, 496, L25

Życki, P. T., Krolik, J. H., Zdziarski, A. A., Kallman, T. R. 1994, ApJ', 437, 597 
TABLE 1

Summary OF the Binary Parameters For GRS 1124-68 AND A 0620-00

\begin{tabular}{|c|c|c|}
\hline Source Name & GRS $1124-68^{\mathrm{a}}$ & A0620-00 \\
\hline Orbital Period, $P_{\text {orb }}(\mathrm{hr})$ & 10.4 & $7.75^{\mathrm{e}}$ \\
\hline Mass Function, $f\left(M / M_{\odot}\right)$ & $3.01 \pm 0.15,3.34 \pm 0.15^{\mathrm{b}}$ & $2.91 \pm 0.08^{\mathrm{d}}, 2.72 \pm 0.06^{\mathrm{f}}$ \\
\hline Companion Type & $\mathrm{K} 3-5 \mathrm{~V}$ & $\mathrm{~K} 5 \mathrm{~V}$ \\
\hline Black Hole Mass, $m=M / M_{\odot}$ & $6.0_{-1.0}^{+1.5}, \quad 5.8_{-2}^{+4.7 \mathrm{c}}$ & $10 \pm 7^{\mathrm{g}}, 4.6 \pm 1.0^{\mathrm{h}}$ \\
\hline Mass Ratio, $q=M_{c} / M$ & $0.133 \pm 0.019, \quad 0.128 \pm 0.04^{\mathrm{b}}$ & $0.067 \pm 0.01^{\mathrm{f}},>0.094^{\mathrm{h}}$ \\
\hline Inclination, $i$ & $60_{-6^{\circ}}^{+5^{\circ}}, \quad 54_{-15^{\circ}}^{+20^{\circ} \mathrm{c}}$ & $37_{-3^{\circ}}^{+28^{\circ} \mathrm{g}, \mathrm{k}} 67 \pm 4^{\circ \mathrm{h}}$ \\
\hline Outer Disk Radius ${ }^{1} R_{\text {out }}\left(10^{11} \mathrm{~cm}\right)$ & $1.9 \pm 0.16$ & $1.2 \pm 0.27$ \\
\hline Distance, $d(\mathrm{kpc})$ & $5.0 \pm 1.1$ & $1.05 \pm 0.4^{\mathrm{g}, \mathrm{m}}$ \\
\hline
\end{tabular}

${ }^{1}$ Computed using equation (2-1).

REFERENCES. - (a) From EMN and references therein, except where explicitly indicated; (b) Casares et al. 1997: (c) Shahbaz et al. 1997; (d) Orosz et al. 1994; (e) McClintock \& Remillard 1986; (f) Marsh et al. 1994; (g) Shahbaz et al. 1994; (g) Haswell et al. 1993; (k) Shahbaz et al. 1999; (m) Barret et al. 1996. 